\title{
A Compact Cold-Atom Frequency Standard Based on Coherent Population Trapping
}

\author{
Francois-Xavier Esnault, John Kitching, and Elizabeth A. Donley \\ Atomic Devices \& Instrumentation Group \\ National Institute of Standards and Technology \\ Boulder, CO, USA \\ edonley@boulder.nist.gov
}

\begin{abstract}
We present the status of our cold-atom clock based on coherent population trapping, including the present clock stability and a preliminary evaluation of the three main systematic frequency shifts: the $1^{\text {st }}$-order Doppler shift, the Zeeman shift, and the light shift.
\end{abstract}

\section{INTRODUCTION}

Although the performance of Chip Scale Atomic Clocks (CSACs) [1] based on Coherent Population Trapping (CPT) has improved considerably over the past few years, CSACs suffer from drift caused by buffer-gas collisions and light shifts that limit their timing uncertainty to the level of one microsecond per day at best [2]. Our study aims to significantly reduce the two main sources of drift in CSACs while maintaining a compact physics package such that timing uncertainties of a few nanoseconds per day can be achieved. We aim to reach a frequency stability of $2 \times 10^{-11} \tau^{-1 / 2}$ and an accuracy of $10^{-13}$ in a very small physics package $\left(10 \mathrm{~cm}^{3}\right)$.

\section{EXPERIMENT}

Our clock is based on a compact source of laser-cooled $\mathrm{Rb}$ atoms, which eliminates the buffer gas and associated shifts. The vacuum system has a two-chambered design, with a $2 \mathrm{D}^{+}$ MOT [3] loading a 3D MOT in a differentially pumped configuration. The volume of the vacuum chamber, including the $2 \mathrm{l} / \mathrm{s}$ vacuum pump, is approximately $150 \mathrm{~cm}^{3}$.

To produce a clean, coherent light source for control and minimization of the light shift, we have developed a laser

This work is funded by NIST and DARPA. NIST is an agency of the U.S. government and this work is not subject to copyright. The views, opinions, and/or findings contained in this article/presentation are those of the author/presenter and should not be interpreted as representing the official views or policies, either expressed or implied, of the Defense Advanced Research Projects Agency or the Department of Defense.

(Approved for Public Release, Distribution Unlimited) system with a relatively pure spectrum based on an optical phase lock loop built from two commercial megahertz-broad DFB/DBR laser diodes [4]. So far, we have achieved a phase error variance of $0.35 \mathrm{rad}^{2}$ between the two lasers, which corresponds to $>70 \%$ of the power in the carrier.

We probe the clock transition using CPT in the lin $\|$ lin geometry [5], which is known to produce high-contrast CPT spectra owing to the absence of trap states. The linear polarization of the CPT light can be represented as a sum of $\sigma^{+}$ and $\sigma^{-}$circularly polarized light components, which results in probing the atoms with a superposition of two lambda systems. The relevant energy-level diagram for ${ }^{87} \mathrm{Rb}$ with the two clock lambda systems is shown in Fig. 1.

We use Ramsey interrogation to reduce the average intensity of the CPT light [6]. Typically, we prepare atoms in the dark state with a $300 \mu \mathrm{s}$ CPT pulse with the intensity at $2 \%$ of saturation. Then we let the dark state evolve for an $8 \mathrm{~ms}$ Ramsey time. We then probe the phase of the dark state with a $50 \mu$ s CPT pulse.

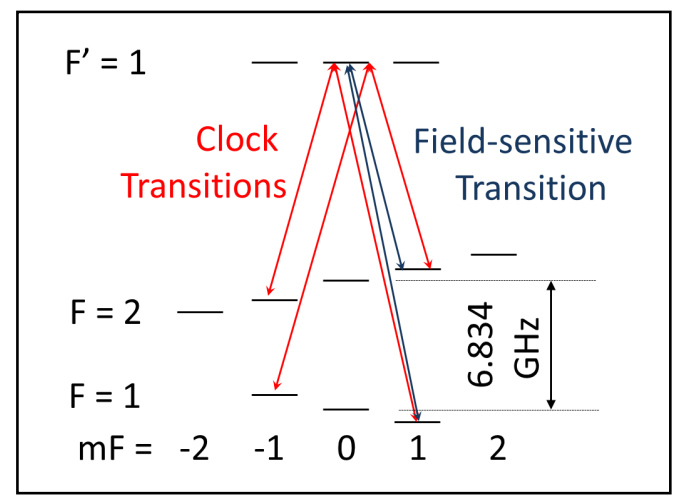

Figure 1. The energy levels of ${ }^{87} \mathrm{Rb}$ relevant to lin $\|$ lin CPT interrogation. The two lambda systems are shown in red. In blue is the field-sensitive transition that we used to measure the magnetic field. 
We limit our interrogation times to less than $10 \mathrm{~ms}$. These short interrogation times allow us to efficiently recapture atoms from cycle to cycle with our $3 \mathrm{~mm}$ MOT beams, enabling a typical laser cooling stage duration of $45 \mathrm{~ms}$ for loading $2 \times 10^{6}$ atoms.

We observe Fourier-limited Ramsey resonances down to a width of $50 \mathrm{~Hz}$ with a transmission contrast of 55\%. To lock the clock to the hyperfine ground state splitting, we use a digital servo to jump the CPT frequency detuning from cycle to cycle to probe the central fringe on opposite sides of the line, and we use the result of the measurements to steer the difference frequency to lock the clock to the central fringe. An Allan deviation of the frequency steers is shown in Fig. 2. The short term stability is currently limited by laser frequency noise to $4 \times 10^{-11} \tau^{-1 / 2}$. The long-term stability is limited by magnetic field drift in our unshielded system, as discussed below.

\section{SYSTEMATIC FREQUENCY SHIFTS}

We have begun to study the influence of systematic frequency shifts on the long-term stability of our clock. The most significant shifts in our system are the $1^{\text {st }}$-order Doppler shift, the Zeeman shift, and the light shift.

\section{A. $1^{\text {st }}$-order Doppler shift}

The $1^{\text {st }}$-order Doppler shift arises from the interaction of moving atoms with the travelling-wave component of our interrogation light. The largest contributor to the atoms' velocity in our system is free fall. With a pure travelling wave and the interrogation light propagating at an angle $\theta$ with respect to the direction of the acceleration of gravity, the $1^{\text {st }}$ order Doppler shift leads to a fractional frequency shift of the clock transition of

$$
\Delta \mathrm{v} / \mathrm{v}_{0}=g T_{R} \cdot \cos (\theta) / c
$$

Here, $g$ is the acceleration of gravity, $T_{R}$ is the Ramsey time, and $c$ is the speed of light.

To initially measure the $1^{\text {st }}$-order Doppler shift in a case where the shift is large, our probe direction is parallel to the direction of gravity. We have probed the falling atoms with travelling waves along and against the direction of gravity and have confirmed the expression in Eq. (1), with the sign of the shift depending on whether the atoms are probed from above or below.

To reduce the shift, we retroreflected the interrogation light. Since our cell does not have an antireflection coating, the CPT interrogation light has standing- and travelling-wave components, with about $14 \%$ of the power in the travelling wave. In the retroreflected geometry, the $1^{\text {st }}$-order Doppler shift is $3.5 \times 10^{-11}$ for an $8 \mathrm{~ms}$ Ramsey time, which is lower by about an order of magnitude than when we interrogate the atoms with a pure travelling wave. Our next-generation vacuum chamber will also be based on an antireflectioncoated cell, which will reduce the power in the travelling wave by a factor of 35 . We can reduce the shift even further by probing the atoms along a direction perpendicular to gravity.

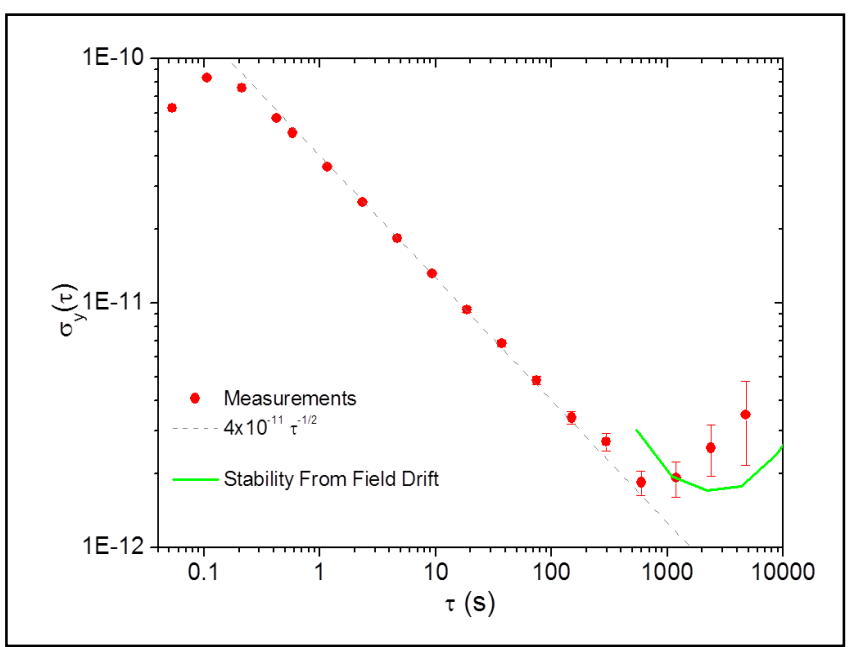

Figure 2. Fractional frequency stability versus interrogation time calculated from an Allan Deviation of the frequency steers. The red points are the measurements. The dashed line is a curve showing a $4 \times 10^{-11} / \sqrt{ } \tau$ dependence.

The green curve is the shift that we would expect to observe from our measured magnetic-field drift, which close to the drift that we would predict from the field measurements.

\section{B. Zeeman Shift}

The two lambda systems that we probe are influenced by first- and second-order Zeeman shifts. Both transitions have the same quadratic sensitivity to magnetic field, but due to the nuclear spin interaction, the two CPT resonances exhibit a weak linear splitting. The overall magnetic shift for the two transitions is given by $\Delta v_{\text {mag }}=575 \mathrm{~Hz} / \mathrm{G}^{2} \pm 2.8 \mathrm{kHz} / \mathrm{G}$. For magnetic shifts smaller than the CPT linewidth, the linear splitting leads to a broadening of the resonance line.

Because of magnetic-field gradients, arising mainly from the ion pump, we are forced to use a bias field that causes a $1^{\text {st }}$-order Zeeman splitting that is significantly larger than the fringe linewidth in order to maximize the fringe contrast. With our Ramsey interrogation and typical magnetic field of 100 $\mathrm{mG}$, the linear splitting exceeds the linewidth by a large factor, and thus only discrete values of the magnetic field lead the two $\Lambda$ systems to interfere constructively.

If the signal strength of the two lambda systems is the same, then the linear Zeeman shift leads to broadening of the CPT resonance and there is no first-order shift. If there is an imbalance, then a first-order shift is possible.

Our current vacuum system is not shielded, and the second-order Zeeman shift currently limits the long-term stability of our clock. We have performed in-situ measurements of the magnetic field where we jump the Raman detuning every 5 minutes while the clock is operating to measure the position of the field-sensitive transition shown in Fig. 1. The field drift currently limits the long-term stability of our clock to the $2 \times 10^{-12}$ level. We expect that by shielding the system with a single-layer magnetic shield, we can reduce the Zeeman shift by a few orders of magnitude. In addition to reducing the effect of magnetic-field drift in our lab, having a magnetic shield will dramatically reduce the magnetic-field 
gradients and allow us to operate the clock with a much smaller bias field.

\section{Light Shift}

As studied previously [7], the interplay between atomic coherence, the light fields, and the multi-level atomic structure leads to complicated light shift behavior, with contributions from resonant, CPT-generating couplings and non-resonant couplings. Because we can precisely control the CPT beam parameters and the atomic internal state, our experiment is a unique test-bed to evaluate the different contributions.

We have observed quite interesting and complex light-shift behavior which we are still evaluating and will reserve for a future publication. We discuss the light shift only briefly here. The measured clock frequency dependence on common-mode laser detuning under our operating conditions is $1 \times 10^{-10} / \mathrm{MHz}$. We control the common-mode frequency detuning of our CPT lasers to the $3 \mathrm{kHz}$ level for 20,000 s integration times, which leads to changes in the light shift of $3 \times 10^{-13}$, which is an order of magnitude smaller than the Zeeman-shift-induced drift in our system on the same time scale.

\section{ACKNOWLEDGMENT}

The authors gratefully acknowledge E. N. Ivanov and T. Brown for valuable design, measurement, and electronics assistance.

\section{REFERENCES}

[1] S. Knappe, V. Shah, P. D. D. Schwindt, L. Hollberg, and J. Kitching, "A microfabricated atomic clock," App. Phys. Lett. 85, 1460-1462, 2004.

[2] R. Lutwak, "The SA.45s chip-scale atomic clock - early production statistics," in 43 $3^{\text {rd }}$ Annual PTTI Meeting, Long Beach, CA, 2011.

[3] K. Dieckmann, R. J. C. Spreeuw, M. Weidemüller,1, and J. T. M. Walraven, "Two-dimensional magneto-optical trap as a source of slow atoms," Phys. Rev. A 58, 3891, 1998.

[4] E. N. Ivanov, F.-X. Esnault, and E. A. Donley, "Offset phase locking of noisy diode lasers aided by frequency division," Rev. Sci. Instrum. 82, 083110, 2011.

[5] A.V. Taichenachev et al., "On the unique possibility of significantly increasing the contrast of dark resonances on the D1 line of ${ }^{87} \mathrm{Rb}$," JETP Lett. 82, 398-403, 2005.

[6] T. Zanon et al, "Observation of Raman-Ramsey fringes with optical CPT pulses," Phys. Rev. Lett., 54, 776-779, 2005.

[7] P. R. Hemmer et al., "AC Stark shifts in a two-zone Raman interaction," J. Opt. Soc. Am. B 6, 1519-1528, 1989.

[8] M. Zhu and L. S. Cutler, "Theoretical and experimental study of light shift in a CPT-based Rb vapor cell frequency standard," in 32nd Annual PTTI Meeting, vol. 311-323 (Reston, Va, 2000). 